\title{
Communications
}

\section{Interactive DICOM Image Transmission and Telediagnosis Over the European ATM Network}

Emanuele Neri, Jean-Philippe Thiran, Davide Caramella, Claudio Petri, Carlo Bartolozzi, Bruno Piscaglia, Benoît Macq, Thierry Duprez, Guy Cosnard, Baudouin Maldague, and Johan De Pauw

\begin{abstract}
The European High-Performance Information Infrastructure in Medicine, $\mathrm{n}^{\circ} \mathrm{B3014}$ (HIM3) project of the Trans-European Network-Integrated Broadband Communications (TEN-IBC) program, started on March 1996 and finished on February 1997, aimed to test the medical usability of the European asynchronous transfer mode (ATM) network in medical image transmission.

The Department of Radiology, University of Pisa, Pisa, Italy, and St-Luc University Hospital, Brussels, Belgium, involved in the project as healthcare partners in the radiological domain, established several connection sessions finalized to test the usability of Digital Imaging and Communication (DICOM) image transmission and interactive telediagnosis tools in the daily radiological practice. The Pisa site was connected to the Italian ATM pilot (Sirius Network) through the Tuscany metropolitan area network (MAN), while St-Luc University Hospital was connected to Belgium ATM network through the Brussels MAN. By means of international connections provided by the European JAMES project, a link between the two sites was established, connecting both national ATM networks. Due to the large variety of hardware present in the medical centers, multiplatform software tools were used and tested: central test node (CTN) release 2.8 [3], VAT [6], NV-3.3 [7], and IDI (UCL homemade multiplatform teleradiology tool for interactive visualization and processing of DICOM images).

During the telediagnosis session, lead by radiologists in both hospitals, each site submitted neuroradiological clinical cases to the other for remote consultation. The connection, available for a period of two weeks, at 2-Mbit/s bandwidth, allowed the transmission of MR images $(256 \times$ $256 \times 12$ bit) and simultaneous multimedia interactive discussion of the cases. Both off-line transmission and review of the images, using the CTN DICOM transfer routines, and on-line interactive image discussion, using the IDI telediagnosis software, were tested successfully from the technical and medical point of view.
\end{abstract}

\section{INTRODUCTION}

The Digital Imaging and Communications in Medicine (DICOM) standard was developed from 1990 to 1996, mainly by the American College of Radiology (ACR) National Electrical Manufacturers Association (NEMA) committee in the United States, with contributions from European standardization organizations [Comité Européen de Normalization-Technical Committee 251 (CEN-TC 251): Medical Informatics], the Japanese Industry Radiology Apparatus (JIRA), the IEEE, Health Level 7 (HL7) and the American National Standards Institute (ANSI) as well as from European manufacturers and societies [1]. It has been widely accepted as a European standard

Manuscript received November 10, 1997; revised January 29, 1998.

E. Neri, D. Caramella, C. Petri, and C. Bartolozzi are with Diagnostic and Interventional Radiology, Department of Oncology, University of Pisa, 2-I56121 Pisa, Italy (e-mail: neri@ do.med.unipi.it.).

J.-P. Thiran, B. Piscaglia, and B. Macq are with the Laboratoire de Télécommunications et Télédétection, Université Catholique de Louvain, B-1348 Louvain-la-Neuve, Belgium.

T. Duprez, G. Cosnard, and B. Maldague are with the Radiology and Medical Imaging Department, St-Luc University Hospital, Université Catholique de Louvain, B-1200 Brussels, Belgium.

J. De Pauw is with MID-SIM, B-1080 Brussels, Belgium.

Publisher Item Identifier S 1089-7771(98)03691-7.

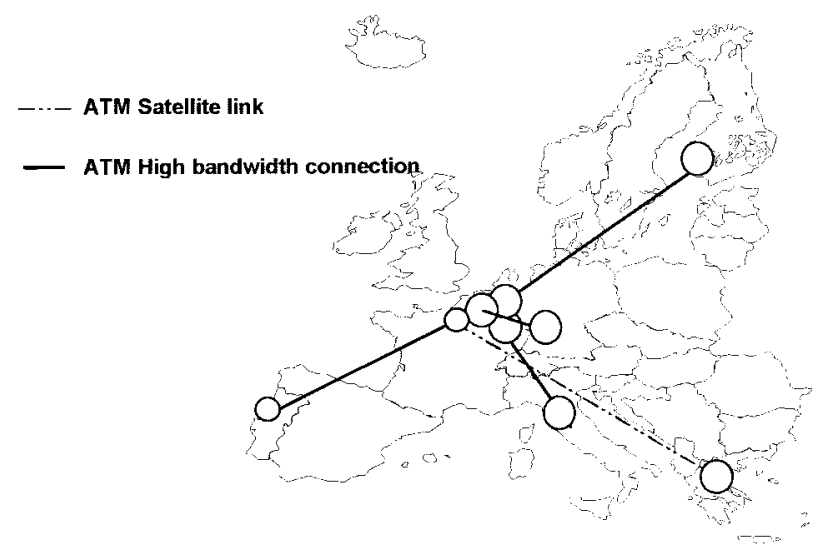

Fig. 1. Geographical situation of the HIM partners.

under the name MEDICOM. This standard allows the exchange of medical images and related information between systems from different manufacturers. The main definitions that are offered by this standard are the format of the image data, the structure of the so-called header containing information about the images from the acquisition phase of the image data (e.g., patient name, resolution, and many other fields), and the so-called service classes that lead the exchange of the images and data. One of the advantages of DICOM is that only part of the defined keys are prespecified (and hence standardized). This leaves the possibility to add user-defined or application-specific information to DICOM images, especially with respect to developing software that deals with header and key information in a user-friendly and architecturally independent way.

We describe the use of the DICOM/MEDICOM standard for image communication within the European Union project High-performance Information infrastructure in Medicine, project $n^{\circ}$ B3014, third extension (HIM3). In this project, the use of DICOM was limited to remote file transfers from image servers accessed via an asynchronous transfer mode (ATM) backbone. The participants selected and transferred images to their own DICOM compatible viewing stations (after access rights had been granted) on the basis of the public domain central test node (CTN) 2.8 DICOM image transfer package. In a second step, interactive telediagnosis has been implemented and assessed by using a multiplatform telemedicine package called IMIS, developed by one partner of this project (Université Catholique de Louvain).

\section{Materials AND Methods}

The Department of Radiology, University of Pisa, and St-Luc University Hospital, involved in the HIM3 project as healthcare partners in the radiological domain, established several connection sessions over the European ATM network to assess the usability of DICOM image transmission and interactive telediagnosis tools in the daily radiological practice.

\section{A. HIM3 Project of the European Union}

The European HIM project of the Trans-European NetworkIntegrated Broadband Communications (TEN-IBC) program, sponsored by the European Commission, DG XIII, started on March 1996 and finished on February 1997, was aimed to assess the medical usability of the ATM international network in medical image transmission and telediagnosis. 


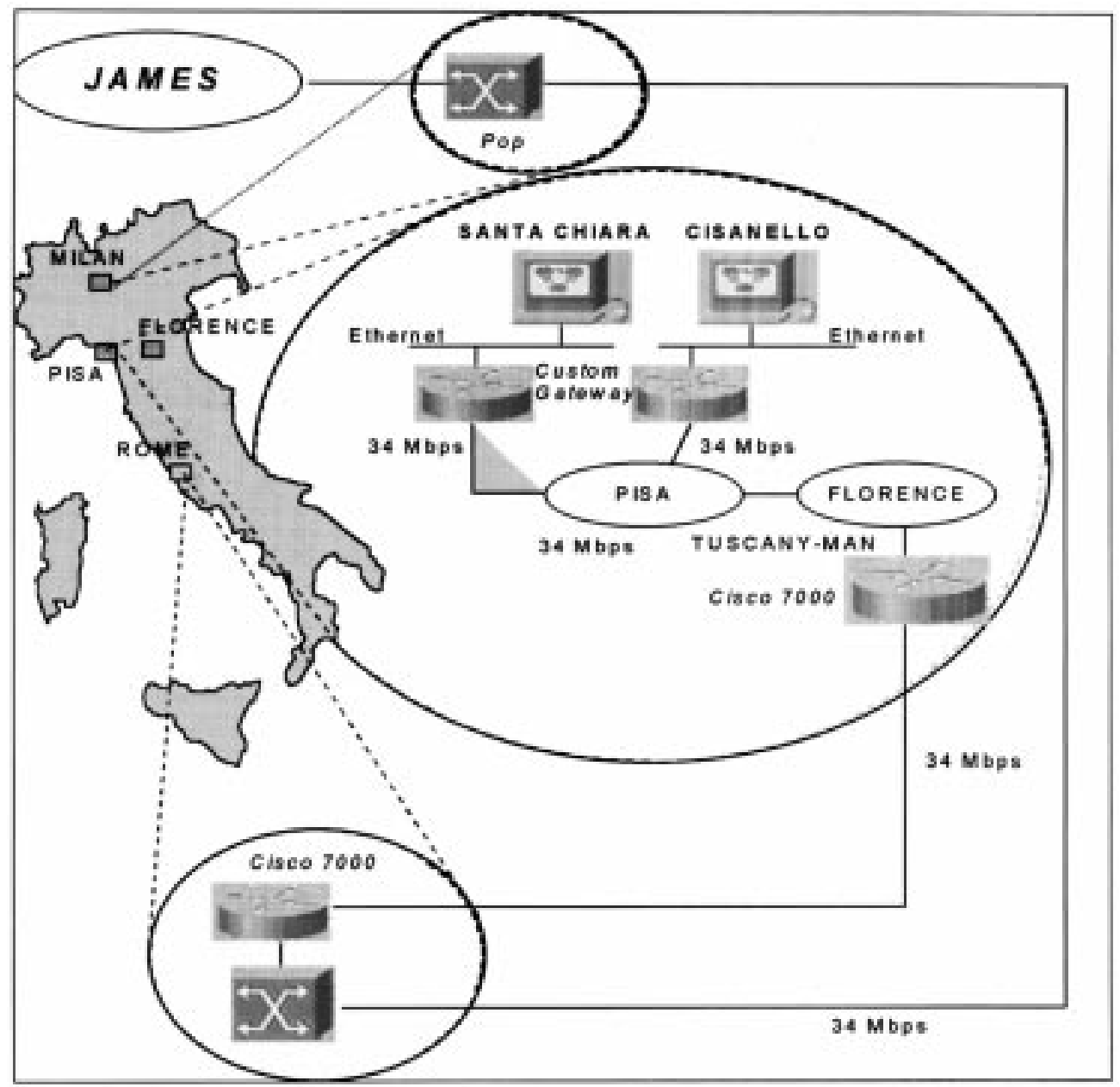

Fig. 2. Network description of the Pisa site.

The HIM project resulted in an ATM network between the medical partners, in which international links were provided by another European project called "JAMES." Fig. 1 presents a map of this network with the geographical situation of the connected partners. Each participating country was connected by a Point-of-Presence (PoP) to the international ATM network.

On the basis of this network, several telemedicine scenarios have been defined, namely, telecardiology, cooperative medical image processing, tele-education, and teleradiology.

\section{B. Teleradiology Scenario}

The radiological field is characterized by the management of digital images and, consequently, needs fast networks and fast computers for image management and processing.

In many modalities, such as magnetic resonance (MR) and computed tomography, images are directly digitally produced and can only be managed by using computers; nowadays, computers provide sufficient resources to efficiently manage digital data.

In the HIM3 project, MR images acquired with different scanners, in Pisa with a 1.5-T unit (Signa, General Electric Medical Systems, Milwaukee, WI), in Brussels with a 0.5-T unit (GiroScan, Philips Medical System, Best, The Netherlands) and with a 1.5-T unit (Signa, General Electric Medical Systems, Milwaukee, WI), were transmitted through the European ATM network for the purpose of discussing neurological cases. Two radiologists, one in Pisa experienced in abdominal and computer applications of radiology (D.C.) and one in Brussels experienced in neuroradiology (T.D.), discussed some examinations in real time and relative to the daily routine. In fact, while in Pisa (the neurological field was a minor part of the diagnostic activity), the integration of the experience provided by the neuroradiologist in Brussels appeared to be very helpful in reaching the final diagnostic decision.

\section{Pisa Site}

Two city hospital sites (Santa Chiara and Cisanello, both in Pisa) were connected to the Tuscany metropolitan area network (MAN), which consists of two 34-Mbps DQDB-MAN's, one in Pisa and the other in Florence, Italy. Both MAN's were interconnected and were linked over a router in Florence to the ATM switch in Rome, Italy, which on this turn is connected to the switch in Milan, Italy, as shown in Fig. 2.

The connection between the hospital's own local area networks (LAN's) (Ethernet) and the Tuscany MAN was realized by a Custom Gateway of Siemens, which makes the following conversion:

$$
\begin{aligned}
& \begin{array}{ll}
\text { TCP } & \text { TCP } \\
\text { IP to IP }
\end{array} \\
& 802.3 \quad 802.6
\end{aligned}
$$

The Cisco 7000 router provides access to the ATM network by converting incoming DQDB slots into ATM cells and vice versa. This results in the following protocol stack:

$$
\begin{gathered}
\text { TCP } \\
\text { IP } \\
\text { LLC/SNAP }
\end{gathered}
$$

AAL 5. 


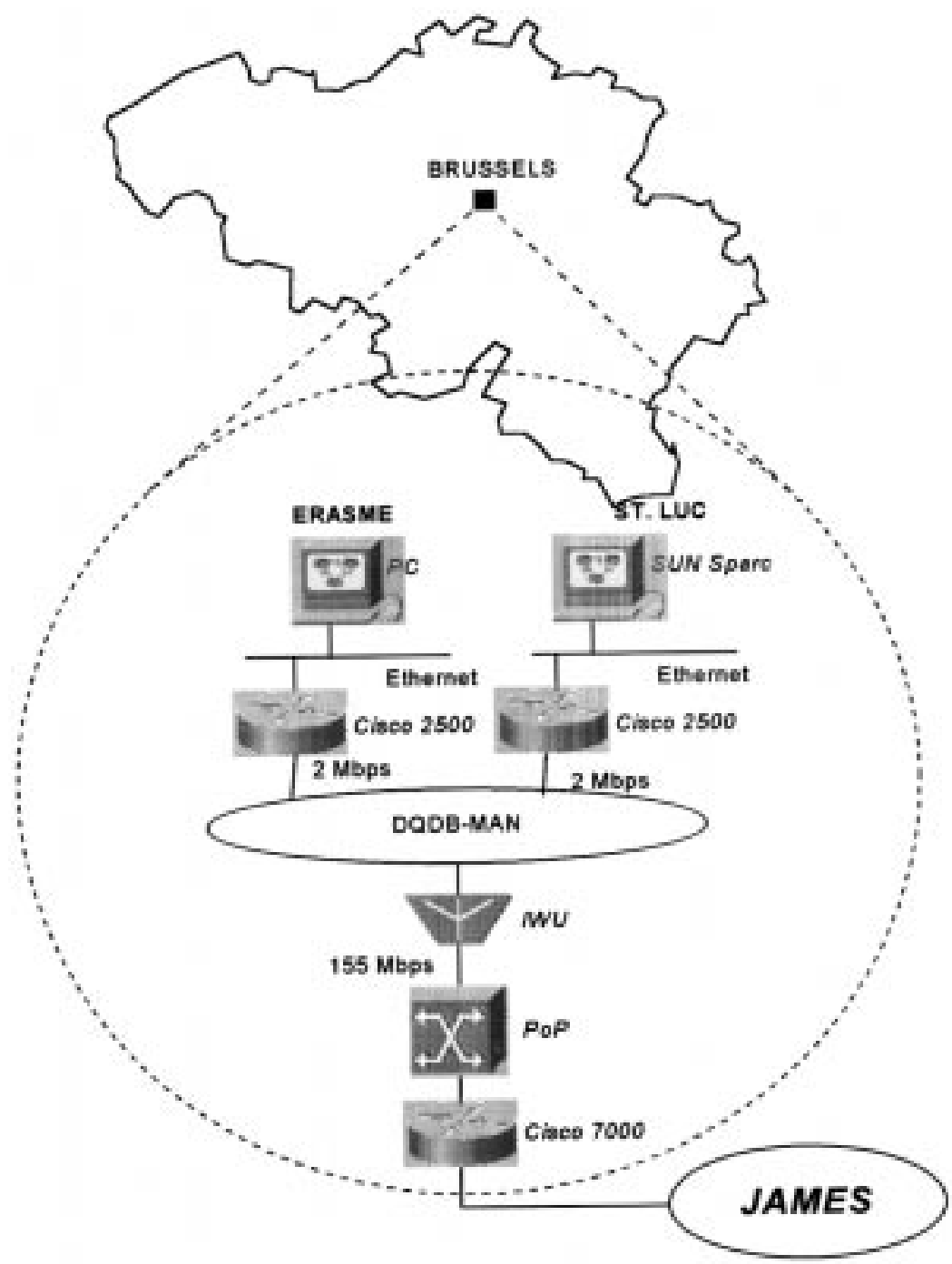

Fig. 3. Network description of the Brussels site.

The hardware installed at the Department of Radiology, University of Pisa Hospital, was made out of SUN SPARC workstations and of Silicon Graphics Indy workstations, both running a UNIX operating system. They were connected to the DQDB Tuscany MAN through the hospital internal Ethernet network, as described in Fig. 2.

\section{Brussels Site}

The St-Luc University Hospital was connected to the Belgian JAMES PoP, also situated in Brussels, through the Brussels DQDB MAN, as illustrated in Fig. 3.

The Brussels sites all have their own LAN (Ethernet), which was connected to the Brussels DQDB-MAN (140 Mbps) by means of a Cisco 2500 router. The DQDB nodes were located in the hospitals. The routers convert as follows:

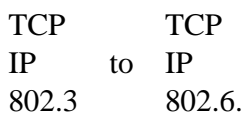

The MAN-Interworking Unit (IWU) with a DQDB Line Interface Module (LIM) provides access to the ATM network. The LIM converts incoming DQDB slots into ATM cells and vice versa. No routing function will take place, resulting in the following protocol stack:

TCP
IP
802.6
AAL $3 / 4$.

Finally, there is a Cisco 7000 router near the PoP to make a AAL3/4 to AAL5 conversion. The hardware installed at the St-Luc University Hospital was made of SUN SPARC workstations.

Let us notice that the security aspects of the network connections, in terms of access control to the hospital networks, from outside were treated by each hospital under its own responsibility and were outside the scope of the project. For instance, at St-Luc's, the network connection to the hospital LAN was done through a Firewall computer.

\section{E. Software}

In this set of trials related to teleradiology, two kinds of experiments have been done. First, we have tested image transfer using the well-established DICOM protocol. Then we have run into interactive telediagnosis tests and practice.

In the first phase, simple medical image transmission has been used: MR image studies have been transmitted from one site to the other using a standard DICOM implementation package called CTN release 2.8 [2], [3]. DICOM images were exported from the scanners 


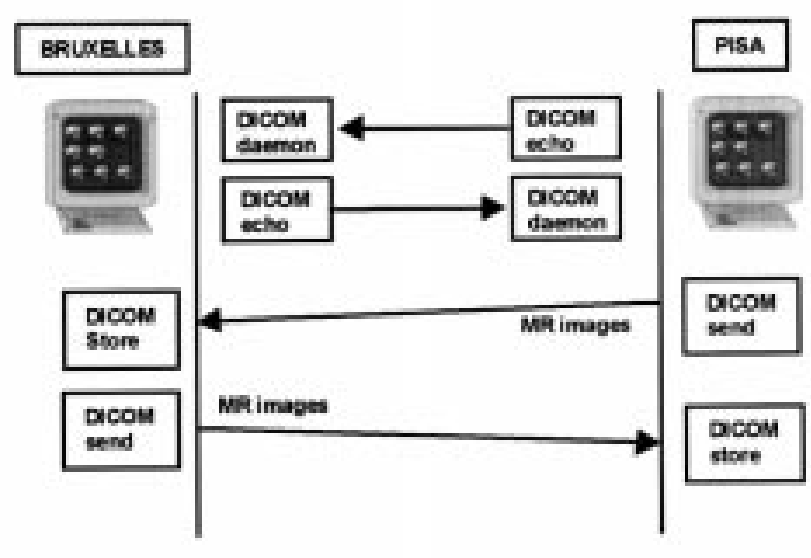

Fig. 4. Trial scheme: DICOM transmission of MR images.

DICOM database to a local CTN database and then sent to the remote site using the CTN send routines, as shown in Fig. 4. On both sites, the images were visualized using their usual clinical visualization systems. Comments on these studies were reported off-line.

In the second phase, interactive telediagnosis sessions were established using a multiplatform teleradiology software package, called IMIS [4], [5], and developed in the Telecommunication Laboratory of the Université Catholique de Louvain, Brussels, Belgium, a partner in the HIM Project. This package allows us to receive medical images from a local DICOM database. These images are then loaded into the IMIS software and sent to a remote IMIS system running on the other site. The current study is then displayed on the two sites, and interactive telediagnosis is facilitated by several functionalities included in this system, such as the use of a shared pointer, allowing us to point and draw regions of interest on the images, and the use of interactive window levelling.

The IMIS software package has been developed as a multiplatform system, particularly well suited for the use in an hospital environment, characterized by the heterogeneity of its computer materials. IMIS makes use of the Tcl/Tk programming language, allowing this to run on a large number of hardware and operating system environments, such as UNIX, Microsoft Windows95, NT, and Macintosh. It also includes advanced compression and signal processing facilities that have not been used in these trials.

This software has been coupled with an audio-video system for conferencing, made of freeware software available on the Internet for various hardware platforms: VAT (an audio conferencing tool from Lawrence Berkeley National Laboratory [6]) and NV-3.3: (video conferencing tool from Xerox Parc [7]).

Together with IMIS, these two A/V tools allowed a real interactive audio-visual telediagnosis over the ATM high-speed network.

\section{RESULTS}

During the telediagnosis session, lead by radiologists in both hospitals, each site submitted neuroradiological clinical cases to the other for remote consultation. The connection, available for a period of two weeks, at 2-Mbit/s bandwidth, allowed the transmission of MR images $(256 \times 256 \times 12$ bit $)$ and simultaneous multimedia interactive discussion of the cases. Both off-line transmission and review of the images, using the CTN DICOM transfer routines, and on-line interactive image discussion, using the IMIS telediagnosis software, were tested successfully from the technical and medical point of view.
ATM provided efficient (fast) transmission of medical images, despite the huge amount of data. The transmission rate ranged between $0.6 \mathrm{Mbit} / \mathrm{s}$ and $1 \mathrm{Mbit} / \mathrm{s}$ (mean $974 \mathrm{Kbit} / \mathrm{s}$ ), changing over time of the connection. The integrity of DICOM images transmission (no data loss: 0\%) was respected.

A public DICOM implementation (CTN) allowed the export, transmission, and remote management of multivendor and multimodalities images. However, the interface provided by this software was not well accepted by the radiologists. In the case of IMIS software, audio and video for remote interactivity were of acceptable quality. The hardware platform did not represent a key factor for all software implementations.

Despite these advantages, there were some limiting factors, such as the cost of the connections and the fact that our experience with the connections was not on demand, rather, it had to be requested through bureaucratic ways. For a local connection from the University of Pisa to the Italian ATM PoP, the cost was $2 \mathrm{ECU}$ for Mbit/h. Eight hours of connection were charged 32 ECU.

\section{CONCLUSION}

ATM, Internet, and multimedia applications were the mixture of our experience. These different components, used in an integrated environment, provided an important impact on the patients and work management, which must be taken into account in future applications.

Teleradiology is characterized by a huge amount of digital information exchanges. This situation is much more evident when long distances have to be covered, such as in the international links. On the basis of our experience, we think that the potentialities offered to the radiological field by the ATM technology, enhancing the quality of transmission, will encourage the development of much more focused applications that could be introduced in the routine clinical practice. This project has clearly shown not only that ATM is technically efficient for multimedia communications, but also that broadband communications do present a real interest from the medical point of view [8]-[11].

\section{REFERENCES}

[1] For details on the DICOM standard, see the DICOM WWW home page: http://www.xray.hmc.psu.edu/dicom/dicom_home.html.

[2] S. M. Moore, S. A. Hoffman, and D. E. Beecher, "DICOM shareware: A public implementation of the DICOM standard," in Medical Imaging 1994-PACS: Design and Evaluation, R. Gilbert Jost, Ed., in Proc. SPIE 2165, 1994, pp. 772-781.

[3] The CTN package is available on the following ftp site: ftp.erl.wustl.edu.

[4] J.-P. Thiran, B. Piscaglia, P. Piscaglia, B. Macq, and R. Demeure, "IMIS: A multi-platform software package for telediagnosis and 3D medical image processing," in Proc. Eur. Conf. Multimedia Applicat., Services, Tech., ECMAST'96, Louvain-la-Neuve, France, vol. 1, pp. 63-75.

[5] J.-P. Thiran, B. Piscaglia, P. Piscaglia, B. Macq, J.-F. Goudemant, and R. Demeure, "IMIS: A multi-platform software package for telediagnosis and 3D medical image processing," in Proc. IEEE ICIP'96, vol. 2, pp. 273-276.

[6] VAT is available on the following ftp site: ftp.ee.lbl.gov.

[7] $\mathrm{NV}$ is available on the following ftp site: parcftp.xerox.com/pub/netresearchv-3.3beta.

[8] E. Neri, D. Caramella, F. Trincavelli, C. Petri, M. Del Sarto, and R. Dell'Osso, "Radiological DICOM images transmission over the European ATM network," Eur. Radiol. 1997, suppl. 7:17.

[9] D. Caramella, E. Neri, M. Del Sarto, R. Dell'Osso, F. Trincavelli, and C. Bartolozzi, Intra-institutional teleradiology: 5 years experience, RSNA 96.

[10] D. Caramella, "State of the art in teleradiology: Teleconsulting and telereporting," Eur. Radiol. 1997, suppl. 7:105.

[11] D. Caramella, R. Lencioni, S. Mazzeo, and C. Bartolozzi, "Transmission of radiological images using broadband communications," Eur. Radiol. 1994, vol. 4, pp. 377-381. 